\title{
EFFECT OF METEOROLOGICAL FACTORS ON RESPIRATORY SYSTEM DISEASES IN FUNAN, CHINA
}

\author{
ZHAO, X. Y. ${ }^{1}-$ ZhANG, Y. ${ }^{1}-$ FAN, G. C. ${ }^{2}-$ LI, Y. P. ${ }^{3}-$ YIN, CH. Y. ${ }^{1}-$ CHEN, J. Y. ${ }^{*}$ \\ ${ }^{I}$ Department of Neurology, the General Hospital of the People's Liberation Army \\ Beijing, China \\ ${ }^{2}$ News Office of Lanzhou Public Security Bureau \\ Lanzhou, China \\ ${ }^{3}$ Department of Neurology, the First Affiliated Hospital of Zhengzhou University \\ Zhengzhou, China \\ ${ }^{4}$ Health Bureau, Logistics Support Department of the Central Military Commission \\ Beijing, China \\ *Corresponding author \\ e-mail:kkn2oa@163.com
}

(Received 19 $9^{\text {th }}$ Apr 2019; accepted 17 $7^{\text {th }}$ May 2019)

\begin{abstract}
To explore meteorological and environmental impacts on human health, a distributed lag non-linear model and a generalized additive model were employed to study the exposure-response relationship between meteorological factors and respiratory system diseases from 2013 to 2016 in Funan, China. The results showed that the decline in patient numbers occurs in the high temperature seasons. At a short time lag, high temperature is a risk factor, but high temperature reduces the number of patients with respiratory system disease at long time lags. Low relative humidity $(\mathrm{RH})$ increases the risk of respiratory system diseases, and largescale 48 -hour temperature changes $(\Delta \mathrm{T} 48)$ increase the risk of disease for a whole range of lags. There is a remarkable correlation between morbidity and meteorological factors, with temperature minima corresponding to peaks in the number of patients. For low RH, the lag effect is obvious for the first 4 days; for higher $\mathrm{RH}$, the effect is weak. A back-propagation artificial neural network model constructed in this study was capable of effectively predicting respiratory system disease, using average temperature, $\Delta \mathrm{T} 48, \mathrm{RH}$, and maximum wind speed as inputs.
\end{abstract}

Keywords: temperature, relative humidity, respiratory system diseases, generalized addictive model, distributed lag nonlinear models

\section{Introduction}

Due to their high morbidity and difficulty of prevention, respiratory system diseases are currently the fourth leading cause of death in Chinese cities (13.1\%), and the third leading cause of death in villages (16.1\%) (Chakraborty et al., 2014). Prevention of respiratory system diseases is still unsolved. From the perspective of disease prevention, meteorological factors are uncontrollable. There is no doubt, however, that predictable meteorological conditions affect the risk rate (RR) and criticality of respiratory system diseases to some degree, although the relevant research is quite limited (Winkelstein et al., 1968; Kim et al., 1996).

Respiratory system diseases are common and frequently occurring diseases that have been studied for a long time. In the international classification of diseases ICD-10 (a coding of diseases and signs, symptoms, abnormal findings, complaints, social circumstances, and external causes of injury or disease), beside diseases of the full 
respiratory system (J00-J99), there are two other subclasses, upper respiratory tract infections (URI) and lower respiratory tract infections (LRI) (Miyamoto et al., 2008; D'amato and Cecchi, 2008). Bull and Quayle (1982) argued that respiratory system diseases are affected by many factors, including but not limited to nutrition intake, climatic variation, and air quality. Ricciardolo et al. (2004) pointed out that the correlation between air contaminants (such as nitric oxide) and respiratory system diseases is strong, but that climate change is certain to affect air contamination even though no relevant research exists. Barreca (2012) found that extremely humid conditions increase the morbidity of respiratory system diseases; however, compare with extreme climate conditions, the study of changing trend of regular climate is more useful. Even though, the effects of meteorological factors on respiratory diseases are still unclear. And current knowledge which provided by experimental studies on the relationship is not sufficient (Kunst et al., 1993). Although the aforementioned studies have not fully clarified the relationship between meteorological elements and respiratory system diseases, fortunately, the most influential factors (such as the temperature and relatively humidity) are clearly known. Thus, quantitative research on the relationship between meteorological elements and respiratory system diseases is necessary.

In order to make such research meaningful, a representative sample is necessary. Funan county, situated in southeast China, is about 500 kilometers from the Yellow Sea. Funan has a standard temperate continental monsoon climate (Liu and Ding, 2010), with four distinct seasons. As this village has never been studied before, no urban heat island effect as well as over use of air conditioner, the influence of meteorological factors is more obvious, and differently from a city, the minimal size of the transient population reinforces the connection between patients and meteorological factors. Thus patient data concerning respiratory system diseases in Funan constitute a representative sample.

In this study, to aid in establishing and perfecting the health early-warning system, the relationship between meteorological elements and respiratory system diseases was systematically evaluated. A generalized additive model (GAM, widely applied inthe meteorological, environmental, and health fields) and a distributed lag nonlinear model (DLNM, used to describe the lagged effect of meteorological elements on health) were employed to quantitatively analyze the effects of several meteorological elements. The relative risk (RR) was used to represent the impact of a set of meteorological factors (Holcomb et al., 2001; Hoek et al., 2013; Liang, 2015; Song et al., 2018). Temperature, relative humidity $(\mathrm{RH})$ and heat index $(\mathrm{HI})$ were the primary meteorological factors analyzed. This study hopes to study the temperature and other meteorological elements affect the respiratory system to improve the meteorological factors on he health early-warning system. The results have far-reaching implications for respiratory system disease prevention, not only for Funan County, but also for other regions with similar climate conditions.

\section{Materials and Methods}

\section{Data concerning diseases and meteorological elements}

Funan (Anhui Province, China) has a population of 1.697 million, including 884,000 males and 813,000 females, with a sex ratio of 1.087:1. Statistical data from respiratory disease patients were collected from Jan 1, 2013 to Dec 31, 2016. The data were 391185 cases which included the date of inpatient visits, age, sex, primary diagnosis 
and address. Respiratory disease (ICD-10: J00-J99), upper respiratory disease (J00-J06, J30-J39), and lower respiratory disease (J20-J22, J40-J47) were classified according to the $10^{\text {th }}$ revision of International Classification of Diseases. A total of 50680 people were hospitalized for respiratory diseases, of which 31267 were hospitalized for upper infections and 6942 for respiratory infections.

The meteorological data mentioned above were provided by the China meteorological data sharing service system (http://cdc.nmic.cn/home.do). The data contained daily mean temperature, daily minimum temperature, daily maximum temperature, and relative humidity, were obtained and other variables from the China. The relationship between these meteorological elements and the status of the patients was systematically examined in this study.

\section{Analysis methods}

To quantitatively analyze the effects of different meteorological elements, a generalized additive model (GAM) and a distributed lag non-linear model (DLNM) were used.

A GAM assumes that linear variables and non-linear variables can be combined in the form of a sum, in which the effects of the non-linear variables can be represented by nonlinear function terms (Barnett et al., 2012; Gasparrini et al., 2015; Barr and Zacks, 2017). There are no restrictions concerning dependence relationships between the causal variables, so the flexibility of a GAM is high. A GAM uses a link function to describe the relationship between the mean of the response variable and a smooth function of each explanatory variable (Yee and Mitchell, 1991). The advantage of a GAM is the ability of this type of model to deal with highly non-linear and non-monotonic relationships between the response and the explanatory variables. In this study, smooth functions (thin-plate spline, natural spline, B-spline) were used for fitting the independent variables. The Akaike information criterion (AIC) was used to test the goodness of fit for the time series data. The model can be written as:

$$
\begin{gathered}
\log (n)=s(T e m p)+s(\text { med_age })+s(\text { gender_ratio })+ \\
+s(\Delta T 48, d f=7)+s(R H)+s(\text { Wind_max }, d f=6)+\text { dow }+\alpha
\end{gathered}
$$

where $\mathrm{n}$ is the number of patients; log is used as a link function; $\mathrm{s}()$ denotes the thin-plate spline functions for nonlinear variables; Temp, med_age, sex_ratio, $\Delta \mathrm{T} 48, \mathrm{RH}$ and wind_max represent the temperature, median age, sex ratio, temperature difference during 48 hours ( $\triangle \mathrm{T} 48), \mathrm{RH}$, maximum wind speed, respectively; dow (representing the effect of weekday) is a dummy variable, and arepresents the residuals of the GAM.

The DLNM has long been used to describe the lagged effect of meteorological elements on health outcomes (Gasparrini et al., 2010; Wu et al., 2013; Gasparrini et al., 2014). It uses a cross-basis function to describe a two-dimensional relationship along the dimensions of meteorological element and lag. The choice of cross-basis functions for the meteorological element and lag are independent, so spline or linear functions can be used, while polynomial functions can be used for the lag. The resulting estimates can be plotted using a three-dimensional graph to show the relative risks for both meteorological elements and lags (MacDonald, 2015). Cross-basis functions for the meteorological elements (daily average temperature, HI, RH and so on) were established in this study. These effects were estimated using nonlinear smoothing functions for both dimensions, 
in which a natural cubic spline was used for each meteorological element and a polynomial spline for the lag effect. To avoid singularity of the model, it is necessary to avoid having too many nonlinear terms. In this study, therefore, we examined only one variable for each model (the temperature in model I and HI in model II). Step-wise backwards selection was employed in the models. Also, because the HI is computed from RH and temperature, when we included HI in the model, we excluded the other two variables. Thus, the final model can be written as follows:

For temperature:

$$
\begin{gathered}
\log (n)=c b(T e m p)+s(\text { med_age })+s(\text { gender_ratio })+ \\
+s(\Delta T 48, d f=7)+s(R H)+s(\text { Wind_max, } d f=6)+\text { dow }+\alpha
\end{gathered}
$$

For HI:

$$
\begin{gathered}
\log (n)=c b(H I)+s(\text { med_age })+s(\text { gender_ratio })+ \\
+s(\Delta T 48, d f=7)+s(\text { Wind_max }, d f=6)+\text { dow }+\alpha
\end{gathered}
$$

where $c b$ denotes the cross-basis functions for nonlinear variables, and the other terms are defined as in Equation 1.

We also introduce the risk ratio (RR), defined as the ratio of the probability of disease development in a group exposed to a specified environment to the probability in a non-exposed control group. The RR (representing the risk of casualties caused by a unit change of environmental conditions), which can be calculated using the GAM, was used to quantify the impact of meteorological factors on the respiratory patients. The range of $\mathrm{RR}$ is $(0, \infty) ; \mathrm{RR}=1$ means no connection between exposure to an environmental condition and disease; $\mathrm{RR}<1$ means that that exposure results in a reduction of the incidence of the disease (i.e. exposure is a protective factor); and RR > 1 means the opposite (Wong et al., 1999). The corresponding 95\% confidence interval was calculated using the equation:

$$
95 \% \mathrm{CI}=\exp [(\beta \pm 1.96 \mathrm{SE}) \times \mathrm{dt}]
$$

The analyses were performed using the R-3.3.2 software environment. The "mgcv" program package was used for the GAM, and the "dlnm" program package was used for the DLNM.

Additionally, artificial neural network models trained by back-propagation (BP-ANN), originally inspired by biological neural systems, have been among the most successful algorithms for machine learning. In the disease field, BP has been used widely as a method and also a benchmark for predicting disease incidence. It can be superior to other methods because of its highly flexible ability to model nonlinearities.

BP-ANN model is mainly composed of three parts: an input layer, a hidden layer and an output layer. There are several nodes in each layer mapping the input vector (in the input layer) to the output vector (in the output layer) by a continuingly updating weight vector (in hidden layer). The weight is updated by the each iteration error (the difference between the output value and the real value). In our study, the data in the input layer (4 5 neurons) were selected from those meteorological factors which directly or indirectly 
affect respiratory system diseases determined during model construction. The number of respiratory system diseases was chosen as the output layer in the BP model.

The tansig and purelin were selected as transfer functions in the hidden layer and output layer, respectively. An adaptive training function with momentum was used for network training. This algorithm is able to adjust the learning rate and the incidental momentum automatically to avoid the local minima. Moreover, it accelerates the convergence rate considerably. Therefore, we introduce a BP-ANN model here with the goal of making precise predictions of respiratory system diseases and thereby providing technical support for decreasing diseases and reducing economic loss.

\section{Results}

Summary statistics for respiratory diseases are shown in Table 1. During the period of 4 years covered by this study, the average number of patients with respiratory system disease was about 38 per day (male: 24 per day, female: 14 per day). The number of patients with URI was much less than the number with LRI.

Table 1. Composition ratio of different diseases in respiratory system

\begin{tabular}{c|c|c|c}
\hline Sequence of diseases & Name of disease & Number & Constituent ratio \\
\hline 1 & Tracheitis & 14167 & 27.95 \\
2 & Bronchitis & 12442 & 24.55 \\
3 & Chronic obstructive & 9413 & 18.57 \\
4 & pulmonary diseases & 7669 & 15.13 \\
\hline 5 & Asthma & 4671 & 9.22 \\
\hline
\end{tabular}

The Table 2 shows the composition ratio of different diseases in respiratory system. The top five diseases in respiratory system composition ratio are: tracheitis (27.95\%), bronchitis (24.55\%), chronic obstructive pulmonary diseases (18.57\%), asthma (15.13\%), pulmonary heart disease $(9.22 \%)$. The Table 2 shows the incidence of respiratory diseases was negatively correlated with daily mean temperature, daily mean air pressure and relative humidity, the correlation coefficients are $-0.343,-0.032$ and -0.122 ( $\mathrm{p}<0.01)$, and positively correlated with 48-hour temperature change and wind speed, the correlation coefficients are 0.080 and $0.076(\mathrm{p}<0.01)$, respectively.

Table 2. Spearman correlations between respiratory system disease and meteorological factors

\begin{tabular}{c|c|c|c|c|c|c}
\hline & Patients & Temperature & $\Delta \mathbf{\Delta 4 8}$ & Pressure & Wide & RH \\
\hline Patients & 1.000 & & & & & \\
Temperature & $-0.343^{* *}$ & 1.000 & & & & \\
$\Delta$ T48 & $0.080^{* *}$ & $0.178^{* *}$ & 1.000 & & & \\
Pressure & $-0.032^{* *}$ & $0.121^{* *}$ & $-0.173^{* *}$ & 1.000 & & \\
Wide & $0.076^{* *}$ & $-0.072^{* *}$ & $-0.159^{* *}$ & $0.262^{* *}$ & 1.000 & \\
RH & $-0.122^{* *}$ & $0.269^{* *}$ & $-0.164^{* *}$ & $0.579^{* *}$ & $0.060^{* *}$ & 1.000 \\
\hline
\end{tabular}

Note: ${ }^{*} \mathrm{p}<0.01,{ }^{* *} \mathrm{p}<0.01$ 


\section{Age and sex distribution of respiratory system disease}

For respiratory system diseases, as shown in Fig. 1, the morbidity for male patients was higher than that for female patients $(\mathrm{p}<0.0001)$. The median number of male and female patients were 21 and 12 per day, respectively.

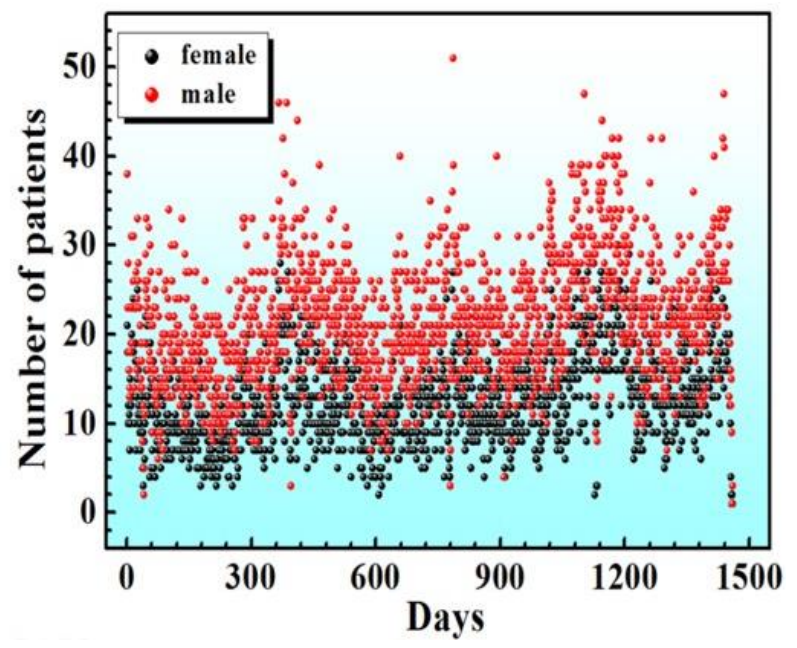

Figure 1. Incidence of respiratory diseases per day, split by sex

The morbidities for different age groups showed marked differences $(\mathrm{p}<0.0001)$, as shown in Fig. 2. For LRI, URI and total respiratory diseases, the peak morbidity appeared for newborns. The number of URI patients decreased as age increased. The trends for LRI and total respiratory disease were very similar, except that a secondary peak occurred for the age range 65-75. During the 5 years studied, the numbers of patients with respiratory system disease were relatively stable; however, the day-to-day variability was remarkable.
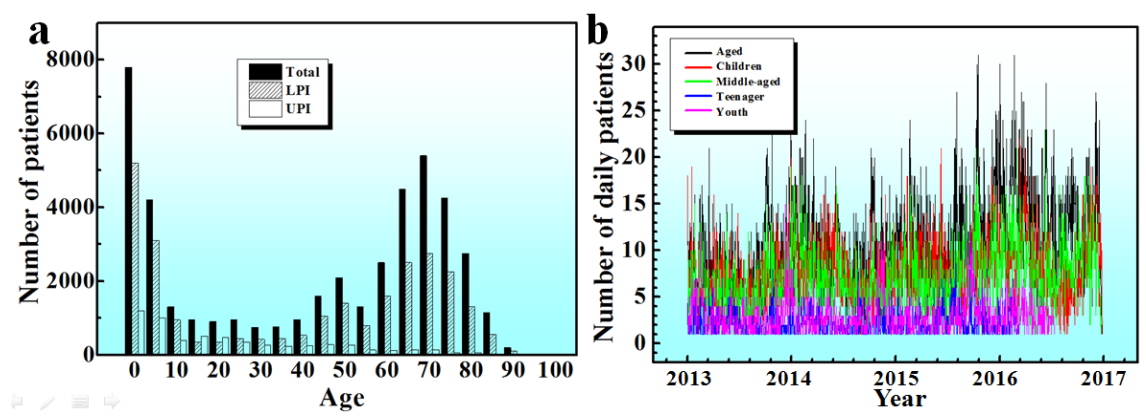

Figure 2. Age distributions and incidences. (a) Histogram showing incidence of upper respiratory tract infections (URI), lower respiratory tract infections (LRI), and total respiratory disease, for each 10-year age group; (b) Number of patients in each age group, for each day

For LRI and total respiratory disease, the occurrence of two peaks can be accounted for as follows: First, infants have lower resistance, their clinical symptoms are more obvious, and they make a larger number of hospital visits than adults. Second, resistance is progressively reduced as people move into old age, while the rate of visits will decrease 
if the patient is too old (more than 75). In other words, the total number of elderly patients is much less than the numbers of young and middle-aged patients. The trend for URI is different, however, because the number of URI patients (compared with LRI or total respiratory disease) is very small, and the accuracy of the statistical data is inadequate. Consequently, in the following sections, the URI is negligible.

\section{Relationship between meteorological elements and respiratory system disease}

Based on the results of the GAM, there was a remarkable correlation between morbidity and meteorological factors such as wind max and $\Delta \mathrm{T} 48$. The relationships between the number of patients each day and various meteorological factors (temperature, HI, RH) are shown in Fig. 3.

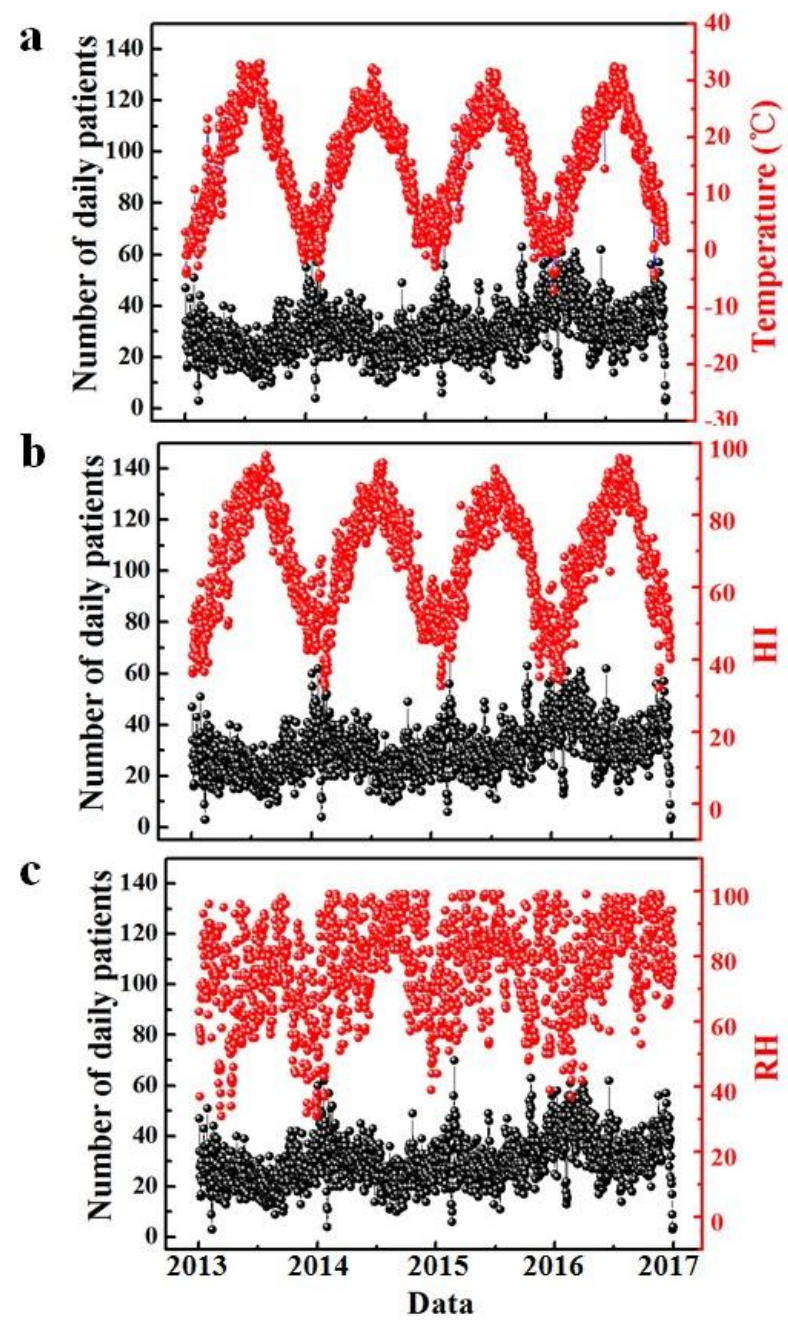

Figure 3. Variations in three meteorological factors, and their relationships with the number of patients. (a) Plot of temperature and number of patients, by day (b) Plot of heat index (HI) and number of patients, by day. (c) Plot of relative humidity and number of patients, by day

The patient number showed a yearly rhythm city, but the amplitude was not very high, with a difference between maximum and minimum of about 20. Meanwhile, because of the continental monsoon climate, the variations of temperature showed a similar 
sinusoidal variation. Minima in temperature corresponded to peaks in patient numbers. A similar relationship was observed between HI and number of patient. For RH, on the other hand, neither early periodicity nor any relationships with the number of patients were obvious.

The relationships between daily patient numbers and season (or month) are presented in Fig. 4. For total respiratory disease, the daily patient numbers for spring and winter were higher, and the number of patients in summer was lowest; the difference between the winter (maximum) and the summer (minimum) was about 30. The 3 months showing the smallest numbers were July, August, and September, corresponding to the summer season. The peak month was January. The distribution as a whole showed a "V" shape. For URI, due to the absence of significance in the statistical data, no systematic results can be stated.
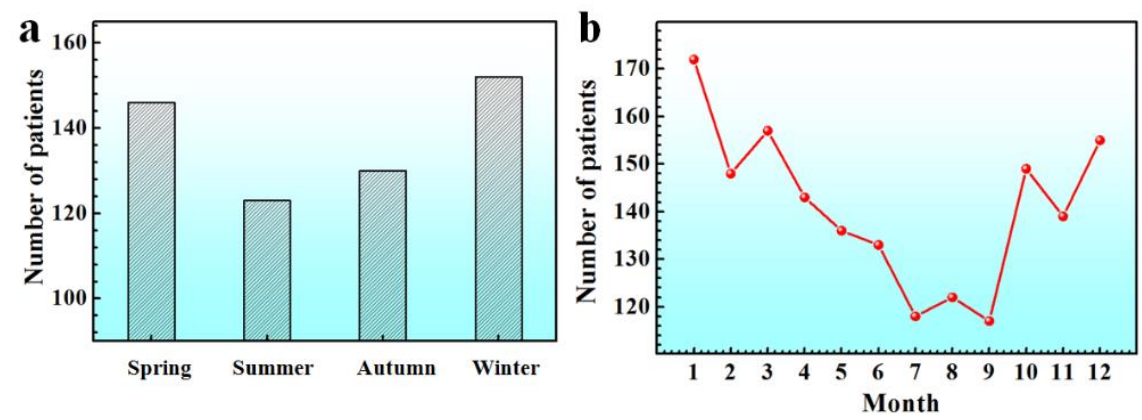

Figure 4. Changes in number of patients as a function of time of year. (a) Histogram showing number of patients for each season. (b) Plot of number of patients for each month of the year

\section{Relationship between meteorological factors and number of patients}

For the DLNM, the effects of sex, age, $\Delta \mathrm{T} 48$ and wind_max were analyzed using the model shown in Equation 2. The maximum of lag employed was 10 days in this study, which was considered sufficient for the current study.

Fig. 5(a) shows a three-dimensional plot of the calculated RR versus temperature and time lag. The maximum RR, corresponding to lags of 0 and 7 days, was 1.05; the minimum RR was 0.92. Examining the contour plot in Fig. 5(b) and the curves in Fig. 5(c), it can be seen that the lag effect of high temperature reached a maximum value at about 4 days; and for low temperature, at about 7 days. An interesting phenomenon can be seen here: low temperature results in the number of patients increasing typically 5 to 9 days after onset, while having protective effects in the first 4 days; whereas for high temperature, the pattern is the opposite, with protective effects for the sixth to ninth days, but harmful effects on the first to sixth days. The analysis also demonstrates a strong protective effect (high RR) of high temperatures on the first day. For a lag of 3 days, high temperature is a risk factor, but for lag of 7 days, low temperature is a risk. By 10 days, almost all temperature effects disappeared. Meanwhile, based on the previous study (Fig. 3), in fact, the relationship among the lag days, patient number and HI is very similar to the relationship, as revealed in Fig. 5. Although, the models for temperature and $\mathrm{HI}$ are not identical, the variation is the same.

Fig. 6(a) and Fig. 6(b) show three-dimensional and contour plots of RR as a function of $\mathrm{RH}$ and lag. For a lag of 3 days, low RH increases the risk of respiratory system disease. The minimum located at the lower left of the Fig. $6(b)$ indicates that for the first day, low 
$\mathrm{RH}$ shows a strong protective effect. For the lower values of $\mathrm{RH}(<50)$, the lag effect is obvious, attenuating after 4 days; for higher RH $(>55)$, the effect of RH on respiratory system diseases is weak $(\mathrm{RR} \approx 1)$. The effect of $\Delta \mathrm{T} 48$ is shown in Fig. $6(c)$ and Fig. $6(d)$. It can be seen that high $\Delta \mathrm{T} 48$ increases the risk of disease for the entire range of lags; lower $\Delta \mathrm{T} 48$ plays a protective role for the first day and for lags of 5-9 days. For values of $\Delta \mathrm{T} 48$ lower than zero, however, no cumulative effect can be observed.
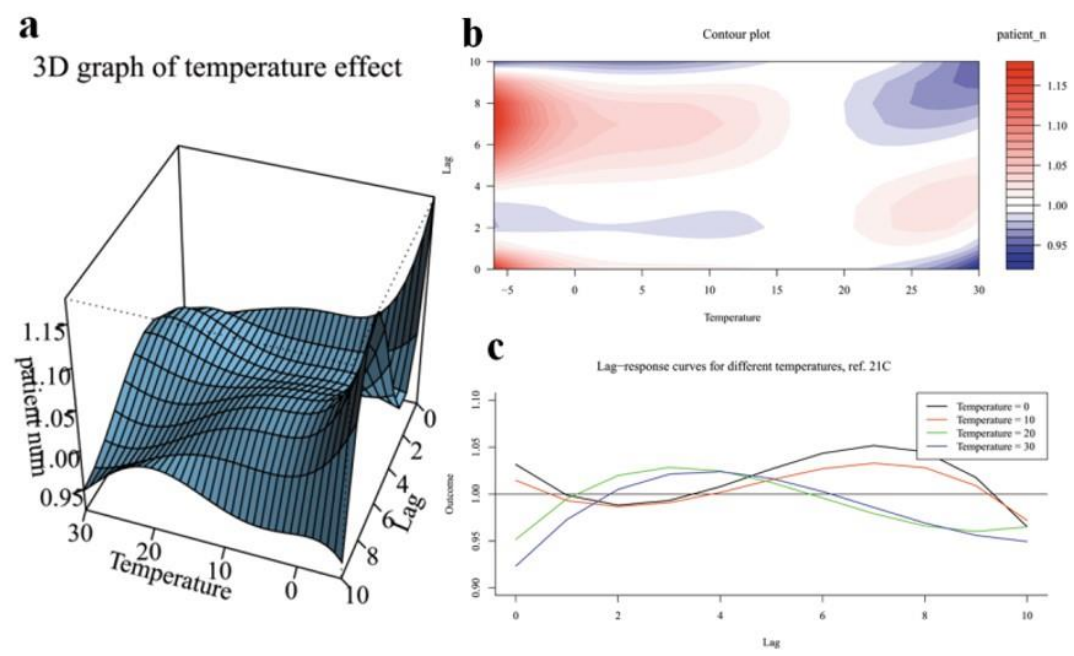

Figure 5. Relative risk (RR) as a function of temperature, for time lags ranging from 0 to 10 days. (a) 3D plot of RR as a function of temperature and lag. (b) Contour map of the same data shown in panel a. (c) Lag-response curves for four different temperatures


Figure 6. Effects of relative humidity $(R H)$ and 48-hour time difference (4T48) on relative risk $(R R)$, for time lags ranging from 0 to 10 days. (a) $3 D$ plot of $R R$ as a function of $R H$ and lag. (b) Contour plot of the same data shown in panel a. (c) $3 D$ plot of $R R$ as a function of $\Delta T 48$ and lag. (d) Contour plot of the same data shown in panel $c$ 


\section{Discussion}

This study investigated the effects of weather on the age and sex distribution of respiratory system disease. Based on the analyses above, it can be concluded that there are strong connections between several meteorological elements and respiratory system disease. When the most important elements, such as temperature, RH, $\Delta \mathrm{T} 48$ and wind-max, are incorporated into a model (all the selected elements are exhibited in Equations (1) to (3), it can be seen that there is a negative correlation between the number of patients and the daily mean temperature (as shown in Fig. 4).

Because the coldest month is January, with the temperature often near or below zero for lengthy periods, the cumulative effect of temperature on respiratory system diseases $\mathrm{s}$ strong; thus it is not difficult to understand why the peak patient numbers appear in January. The main groups at high risk for respiratory system disease in cold temperatures are infants and the elderly. For the elderly, the older they are, the weaker their regulating ability; whereas for infants, their sensitivity is due to underdevelopment. The atmospheric pressure in winter, higher than in summer, can cause labored breathing due to the smaller pressure difference between the human body and the atmosphere. The dynamics of the respiratory system also imply that the exchange of gases inside and outside of the lungs is reduced. Also, cold air and the resulting high pressure and low humidity can combine in irritating the respiratory system. Furthermore, in winter, people spend much more time indoors, thereby increasing the probability of cross infection.

The temperature of February was colder than in March, but the number of patients was smaller. This may be attributable to the spring festival of China, the most important traditional festival, occurring in February. During the spring festival, people do not want to seek medical help except in an emergency, while at the same time the number of available medical workers is reduced. From January to March, the value of $\Delta \mathrm{T} 48$ is positive, which also increases the risk of disease. In summer, the daily high temperature is usually above $30^{\circ} \mathrm{C}$. Although, the cumulative effect can be found in the Fig. 5(c) from the 4 7 lag days. The most important consequence of such temperatures is a protective effect at long lags. The effects of high RH are not particularly strong. For the Funan area, where traditional agriculture is the backbone of the economy, summer is the busiest season for many people; this factor may also reduce the number of patients requesting treatment.

The coldest winter in several years came at the end of 2016 (Fig. 3(a)). At that time, a sudden cold wave struck most of China, including the Funan area, and the number of patients with respiratory system diseases also peaked. This phenomenon is an additional indication that extended cold temperatures are a risk factor.

To further demonstrate the effects of high or low temperatures on respiratory system diseases, the plots in Fig. 7 can be used to examine the RR resulting from heat waves and cold spells, using different temperature thresholds. The threshold for temperature was set at different percentiles of the daily mean temperature range, and durations ranging from 1 consecutive day to 4 consecutive days were analyzed. In Fig. 7(a) and Fig.7(b), the effects of a heat wave on respiratory disease incidence at 0 lag days and 4 lag days are very similar. As the temperature threshold increases, the RR increases, but all the values of RR are less than 1, indicating that heat waves had a consistent protective effect. For cold spells, however, the lag effect is obvious. In Fig. 7(c), at a lag of 7 days, when the threshold temperature percentile is $4 \%$, the RR is 1.58 . The colder the air, the higher the risk ratios, but the $\mathrm{RR}$ is always higher than 1 . The relationship between $\mathrm{RR}$ and $\Delta \mathrm{T} 48$ threshold percentile is shown in Fig.7(d). The RR was basically unchanged after 1 (or 3 
or 4) consecutive days below a given temperature percentile threshold; but for 2 consecutive days, there is an obvious downtrend of RR.

To distribute medical resources efficiently and provide suitable medical care, the prediction of respiratory system disease is indispensable. In fact, a number of prediction methods have been proposed. For example, Liu and Ding (2010) used a multi-principal component regression model to predict the death rate caused by respiratory system disease, yielding a correlation of $\mathrm{r}=0.755$. In this study, a BP-ANN was used to predict the disease; the results are shown in Fig. 8.
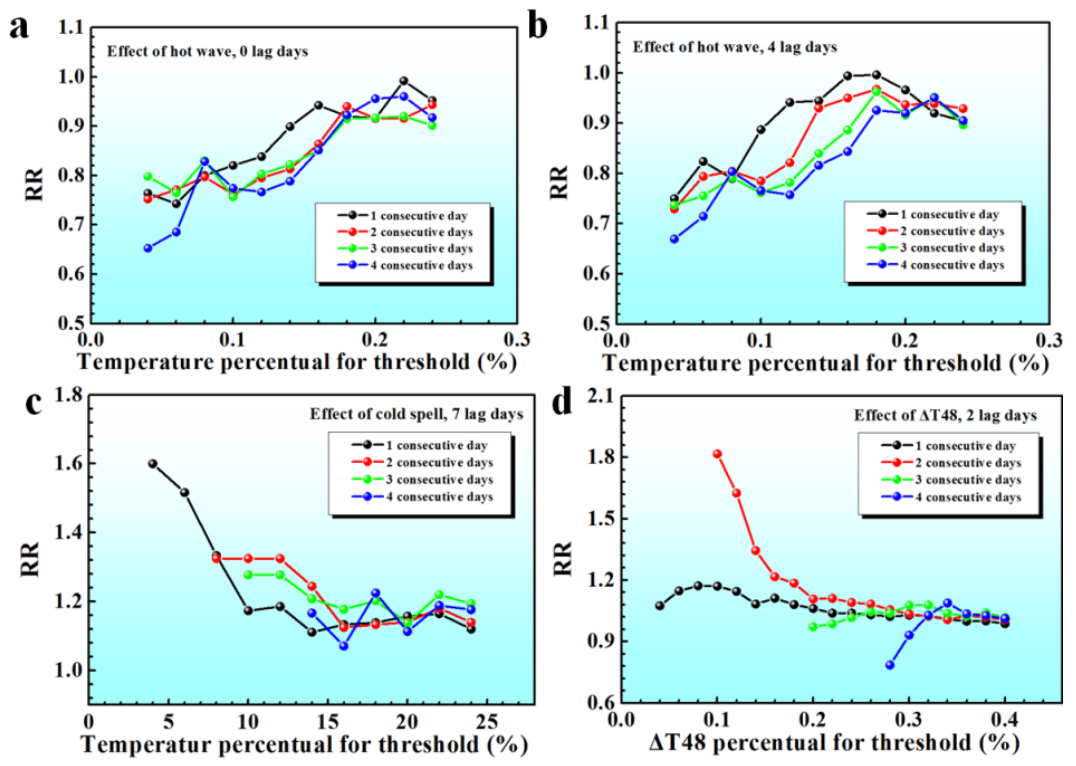

Figure 7. Effects of heat waves and cold spells on relative risk $(R R)$, using different temperature percentiles as thresholds. (a) Effects of heat waves of varying durations, at a lag of 0 days; (b)

Effects of heat waves of varying durations, at a lag of 4 days; (c) Effects of cold spells of varying durations, at a lag of 7 days; (d) Effects of sustained temperature changes of varying durations, at a lag of 2 days
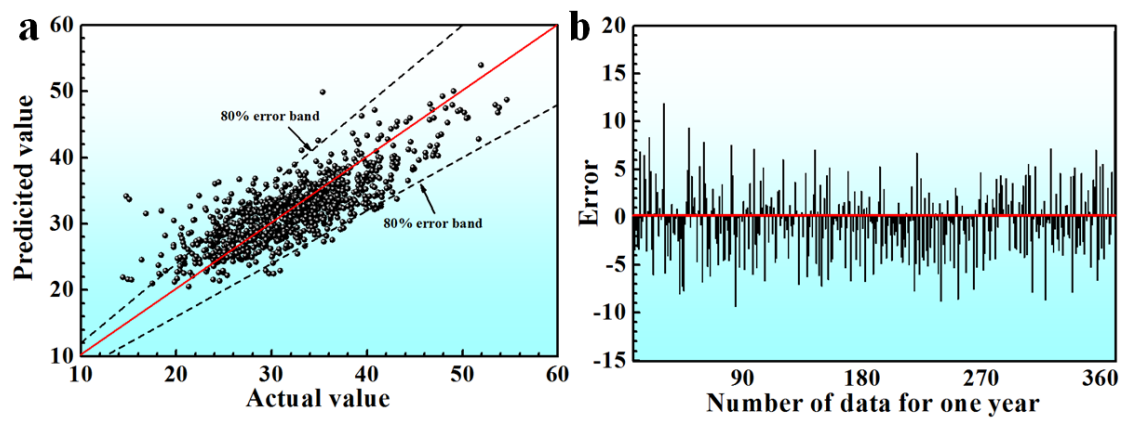

Figure 8. Prediction accuracy of a back-propagation artificial neural network model. (a) Scatter-plot of predicted values versus actual values of numbers of patients; (b) Day-by-day errors over the course of 1 year

Because our investigation of the respiratory system disease was conducted in Funan, a relatively high correlation was obtained. As shown in Fig. 8(a), most of the predicted values are located within the $80 \%$ error band, and the number of positive errors is less 
than that of negative errors. The errors of February and March are slightly higher; this can be attributed to the complex influence factors for those months.

Thus, the BP-ANN model constructed in this work was capable of effectively predicting respiratory system disease, using the average temperature, $\Delta \mathrm{T} 48, \mathrm{RH}$ and wind_max as input data.

\section{Conclusions}

In this study, the distributed lag non-linear model and generalized additive model were investigated to analyze the influence of meteorological factors on respiratory system diseases in Funan, China from 2013 to 2016. Furthermore, a BP-ANN based prediction method for respiratory system diseases has been developed. The main conclusions can be summarized as follows.

During the 4 years, the average number of daily patients of respiratory system disease is about 30; the morbidity of male patients is a little higher than that of female patients, and infants were the highest risk group. There is a remarkable correlation between morbidity and meteorological factors, with temperature minima corresponding to peaks in the number of patients. This may be attributed to a reduction in outdoor activities in winter as well as the influence of cross-infection. For low RH, a lag effect is obvious for the first 4 days; for higher RH, the effect is weak. Air with low RH and low temperature can lead to an increase in the number of patients with respiratory diseases, the weather conditions will dries the mucosa of the respiratory tract and damage sepithelial cells, increasing the susceptibility of the population. High $\Delta \mathrm{T} 48$ increases the risk of disease at all time lags, while low $\Delta \mathrm{T} 48$ decreases the risk at lags of 5-9 days. The BP-ANN model constructed in this work was capable of making an effective prediction of the respiratory system disease, with the main input variables selected being average temperature, $\Delta \mathrm{T} 48$, RH and wind_max. A relatively high correlation was obtained for this prediction method. These developments have conducive to the distribution of medical resources and provision of appropriate medical care, not only in the Funan area, but for the entire monsoon region. In the next step of research, the pathogenic mechanism of meteorological factors on the respiratory effect should by studied, and it will reach a more accurate prediction of the target.

Conflicts of Interests. The authors declare no conflict of interests.

Acknowledgements. This research is supported by the National Key R\&D Program of China (2017YFC1307700).

\section{REFERENCES}

[1] Barnett, A. G., Hajat, S., Gasparrini, A. (2012): Cold and heat waves in the United States. Environmental Research 112: 218-224.

[2] Barr, J. R., Zacks, S. (2017): GLiM: Generalized linear models. - Encyclopedia with Semantic Computing and Robotic Intelligence 1(1): 1630016.

[3] Barreca, A. I. (2012): Climate change, humidity, and mortality in the United States. Journal of Environmental Economics and Management 63(1): 19-34. 
[4] Bull, A. T., Quayle, J. R. (1982): New Dimensions in Microbiology: An Introduction. Philosophical Transactions of the Royal Society B: Biological Sciences 297(1088): 447-457.

[5] Chakraborty, S., Kumar, A., Tiwari, R., Rahal, A., Malik, Y., Dhama, K., Pal, A., Prasad, M. (2015): Advances in Diagnosis of Respiratory Diseases of Small Ruminants. Veterinary Medicine International 2014: 508304.

[6] D'amato, G., Cecchi, L. (2008): Effects of climate change on environmental factors in respiratory allergic diseases. - Clinical \& Experimental Allergy 38: 1264-1274.

[7] Gasparrini, A., Armstrong, B., Kenward, M. G. (2010): Distributed lag non-linear models. - Statistics in Medicine 29(21): 2224-2234.

[8] Gasparrini, A. (2014): Modeling exposure-lag-response associations with distributed lag non-linear models. - Statistics in Medicine 33(5): 881-899.

[9] Gasparrini, A., Guo, Y., Hashizume, M. (2015): Mortality risk attributable to high and low ambient temperature: a multicounty observational study. - The Lancet 386(9991): 369-375.

[10] Hoek, G., Krishnan, R. M., Beelen, R., Peters, A., Ostro, B., Brunekreef, B., Kaufman, J. D. (2013): Long-term air pollution exposure and cardio- respiratory mortality: a review. Environmental Health: A Global Access Science Source 12(1): 43.

[11] Holcomb, J. W. L., Chaiworapongsa, T., Luke, D. A., Burgdorf, K. D. (2001): An odd measure of risk: use and misuse of the odds ratio. - Obstetrics \& Gynecology 98(4): 685-688.

[12] Kim, P. E., Musher, D. M., Glezen, W. P., Rodriguez-Barradas, M. C., Nahm, W. K., Wright, C. E. (1996): Association of invasive pneumococcal disease with season, atmospheric conditions, air pollution, and the isolation of respiratory viruses. - Clinical Infectious Diseases 22(1): 100-106.

[13] Kunst, A. E., Looman, C. W. N., Mackenbach, J. P. (1993): Outdoor air temperature and mortality in the Netherlands: a time-series analysis. - American Journal of Epidemiology 137(3): 331-341.

[14] Liang, S., Saxena, M., Finfer, S., Myburgh, J. (2015): Impact of high temperature on long-term outcomes in patients with traumatic brain injury: A systematic review of observational studies. - Australian Critical Care 28: 42-43.

[15] Liu, W. J., Ding, G. X. (2010): Climate Characteristics of Subtropical High in Western Pacific. - Journal of Anhui Agricultural Sciences 38(16): 8533-8535.

[16] MacDonald, G. (2015): Elements of Time Series Econometrics: An Applied Approach. In: Kočenda, E., Černý, A. (eds.) Economic Record 91(293): 269-270. 2nd edition (Karolinum Press, Prague, 2014).

[17] Miyamoto, T., Kitayama, T., Kumagai, S., Mori, K., Kitamura, S., Shindo, S. (2008): An energy trading system with consideration of $\mathrm{CO} 2$ emissions. - Electrical Engineering in Japan 162: 54-63.

[18] Ricciardolo, F. L. M., Sterk, P. J., Gaston, B., Folkerts, G. (2004): Nitric oxide in health and disease of the respiratory system. - Physiological Reviews 84(3): 731-765.

[19] Song, X. P., Wang, S. G., Li, T. S., Tian, J. H., Ding, G. W., Wang, J. X., Wang, J. X., Shang, K. Z. (2018): The impact of heat waves and cold spells on respiratory emergency department visits in Beijing, China. - Science of The Total Environment 615: 1499-1505.

[20] Winkelstein, J. W., Kantor, S., Davis, E. W., Maneri, C. S., Mosher, W. E. (1968): The relationship of air pollution and economic status to total mortality and selected respiratory system mortality in men: I. Suspended particulates. - Archives of Environmental Health An International Journal 16(3): 401-405.

[21] Wong, T. W., Lau, T. S., Yu, T. S., Neller, A., Wong, S. L., Tam, W., Pang, S. W. (1999): Air pollution and hospital admissions for respiratory and cardiovascular diseases in Hong Kong. - Occupational and environmental medicine 56(10): 679-683. 
[22] Wu, W., Xiao, Y., Li, G., Zeng, W., Lin, H., Rutherford, S., Xu, Y., Luo, Y., Xu, X., Chu, C., Ma, W. (2013): Temperature-mortality relationship in four subtropical Chinese cities: a time-series study using a distributed lag non-linear model. - Science of the Total Environment 449: 355-362.

[23] Yee, T. W., Mitchell, N. D. (1991): Generalized additive models in plant ecology. - Journal of Vegetation Science 2(5): 587-602. 